\title{
HAIR ARTIFACT REMOVAL AND SKIN LESION SEGMENTATION OF DERMOSCOPY IMAGES
}

\author{
JULIE ANN SALIDO, CONRADO RUIZ JR \\ Department of Software Technology, De La Salle University, College of Computer Studies, Manila, Philippines. Email: julie_salido@dlsu. \\ edu.ph , conrado.ruiz@dlsu.edu.ph
}

Received: 08 December 2017, Revised and Accepted: 26 September 2018

\begin{abstract}
Objective: The objective of this research is to perform automatic hair artifact removal and skin lesion segmentation on dermoscopy images.

Methods: Dermoscopy images are images from the examination of the skin lesion using a dermatoscope. There are different types of skin lesion artifacts, structures, or objects that are present in dermoscopy images. This is a pertinent problem that can inhibit the proper examination and accurately segment the skin lesion from the surrounding skin area. Artifacts, such as hair strands, introduce additional features that can also cause problems during classification. Our process starts with hair removal using a median filter on each color space of RGB, a bottom hat filter, a binary conversion, a dilation and morphological opening, and then the removal of small connected pixels. The detected hair regions are then filled up using harmonic inpainting. Then, skin lesion segmentation is performed using a binary conversion, a dilation, a perimeter detection and morphological opening, and then the removal of small connected pixels.
\end{abstract}

Results: Experiments were carried out on the $\mathrm{PH}^{2}$ dermoscopy images. The border of the lesion was quantified for evaluation by four statistical metrics with the lesions identified by the $\mathrm{PH}^{2}$ as the reference image, resulting with a true detection rate (TDR) of 82.31 and a false detection rate of 5.69 .

Conclusions: The results obtained in the research work on hair artifacts removal and skin lesion segmentation provides acceptable results in terms of TDR and low false-positive rates.

Keywords: Artifacts removal, Border detection, Dermoscopy image, Lesion segmentation.

(C) 2018 The Authors. Published by Innovare Academic Sciences Pvt Ltd. This is an open access article under the CC BY license (http://creativecommons. org/licenses/by/4. 0/) DOI: http://dx.doi.org/10.22159/ajpcr.2018.v11s3.30025

\section{INTRODUCTION}

As the population demographics of the world change, it is vital that clinicians are familiar with variations in the prevalence, clinical presentation, and treatment of dermatological disease in people with skin of color. Africans, African-Americans, African-Caribbean, Chinese and Japanese, Native American Navajo Indians, and Hispanics are examples of ethnicities that are considered by dermatologist as persons with skin of color [1].

Clinicians often see patients for the treatment of dermatological disease such as herpes infection, warts, exanthem, urticaria, and insect bites while dermatologists more commonly treat patients for actinic and seborrheic keratosis, bacterial skin infections, psoriasis, and benign and malignant skin tumors [2]. Malignant skin tumors are the most common diagnosed type of skin disease; these can be malignant melanoma and non-melanoma [3]. Cutaneous malignant melanoma (CMM) is the most common disease of people with fair skin. Recently, there has been a rise in incidence even in dark-skinned individuals [4]. Clinically, these appear as a painless, firm, non-tender, and ulcerated skin lesion. Early recognition and treatment of melanoma makes it is almost curable. However, if diagnosed in the later stages, the cancer can progress and spread to other parts of the body and can be deadly. In 2016, it caused a record deaths, an estimated 76,380 are invasive melanomas, and about 46,870 are male and 29,510 are female [5]

Dermatologists and clinicians have striven to diagnose malignant melanoma at the earliest possible stage. The need to attain the goal without inaccurately and needlessly get rid of many benign lesions and other artifacts has steered many techniques that support clinicians in differentiating other skin diseases from MM. Some of these techniques include clinical mnemonics and algorithms, optical imaging instruments, and computer-assisted diagnostic systems which use a dermoscopy. Dermoscopy is used as a non-invasive evaluation [6], in vivo, of color and microstructures of the epidermis, the dermoepidermal junction, and the papillary dermis. These past two decades have opened a new dimension in the examination of pigmented skin lesions (PSLs) and especially in the identification of the early phase of CMM $[7,8]$.

There are several studies in dermoscopy [6] which have developed diagnostic criteria for early detection of melanoma. The studies include ABCD (Asymmetry, Border, Color and Dermoscopic features) rule of dermoscopy [9], and its extension ABCDE (Asymmetry, Border, Color, Diameter and Evolving ) [10], Menzie's scoring method [11], and 7-point checklist [12], and the Ugly Duckling sign for classification of PSL images [13].

The direct application of our work is automatic dermoscopy image classification, which in silico, has four steps: Artifacts and noise reduction, boundary detection, feature extraction, and pattern analysis of lesions. The most challenging tasks are border detection and classification of lesions [14]. For the border detection, usually, image segmentation techniques are used to detect edges of lesions. Moreover, the presence of hair in the images, however, creates a problem during automatic border detection. Automatic hair removal for better segmentation of skin lesion will give a good border detection.

In this paper, we propose a simple method to detect and remove hair from dermoscopy images and then do lesion border detection using image analysis. The hair detection algorithm is tested to $\mathrm{PH}^{2}$ dermoscopy images. The skin lesion border detection was quantified by four statistical metrics with the lesions identified by the $\mathrm{PH}^{2}$ as the ground truth (GT). 


\section{METHODS}

The paper presents an overview of current trend on border detection methods and describes the preprocessing, segmentation, image reconstruction, and lesion segmentation used.

\section{Preprocessing}

The preprocessing phases facilitate the border detection procedure such as color space transformation, contrast enhancement, and artifacts removal. Dermoscopy images are usually acquired with a digital camera attach with a dermoscope. In line with the computational ease and accessibility of scalar processing, the resulting RGB color image is often converted to a scalar image [15].

In dermoscopy, images frequently have artifacts such as ink markings, rulers, air bubbles, and black frames. Some artifacts include intrinsic cutaneous features such as blood vessels, hairs, and skin lines that can affect border detection. Loss of accuracy and an increase in computational time is a result of artifacts and extraneous elements that complicate the border detection procedure. Direct way to remove these artifacts is to even and smoothen the image using a general purpose filter such as the Gaussian (GF) [16], median (MF), or anisotropic diffusion filters [17].

\section{Segmentation}

The process of dividing an image into multiple parts is image segmentation. This is used to recognize objects or other applicable information in digital images. The different means to accomplish image segmentation are using thresholding methods, color-based segmentation, and texture methods [14].

\section{Image completion}

There has been great number of study on image reconstruction, scene completion, or area restoration [18-20]. Image inpainting is the task of restoring a missing part of an image using information from the known part, in this case, the dermoscopy image minus the hair. To repair hairoccluded information from dermoscopy images, a harmonic inpainting method is used for each color space. Moreover, the missing part is the inpainting domain, $D \subseteq \omega$. In the case of harmonic inpainting, where missing part $D$, is filled in by the regularizing term $\Psi(u)$ [21].

$$
\min _{u} \int_{\Omega D}\left(u-u_{0}\right)^{2} d x+\Psi(u)
$$

The fidelity term is used, which is kept to a minimum as in Eq. 1 added with the $\Psi(u)$. The minimization of Eq. 1 is done over an appropriate Banach space $B$ which depends on the choice of the regularizer $\Psi$. The harmonic inpainting $\Psi \Psi(u)=\alpha \int_{\Omega}|\nabla u|^{2} d x$, then $=\mathrm{W}^{1,2}(\Omega) \ldots$.

\section{Lesion segmentation using adaptive thresholding}

Adaptive thresholding algorithm [22] is used for lesion segmentation. It has three steps: Histogram computation, peak detection, and threshold estimation.Convert first input image to gray-level image of channel with the highest entropy based from the paper of Silveira et al. [24]. Then compute the histogram of intensity using the entire pixels inside a rectangular window located at the center of the image. A low-pass filtered using GF filter is used in the histogram. Filter impulse response is,

$G(i)=C \exp \left\{-i^{2} /\left(2 \sigma^{2}\right)\right\}$
Where, $\sigma=5$ and $C$ is a normalization constant. Valley at $i$ is defined as the difference between the straight line defined by the peaks and the histogram amplitude at $i$. If there are more than two peaks, threshold computation of the histogram is used. Then select the significant peaks in the histogram. In the case of two peaks, threshold computation of the histogram is used. The highest peak is selected, if there are a number of peaks. Remaining ones are then tested. In the case of two peaks, maximum peak is selected.

\section{Methods}

This section describes our method from preprocessing of dermoscopy images, to hair removal and inpainting, and to lesion border detection. The flow diagram (Fig. 1) of the lesion border detection on dermoscopy images using image segmentation.

\section{Preprocessing}

Preprocessing is first done on the dermoscopy images of the $\mathrm{PH}^{2}$ dataset [24]. We convert the image into 24 bit Bitmap file. An RGB color space is identified for use and rescaled with $360 \times 450$ image size, to make it uniform to the GT lesion in the dataset. Gray scale and binary image are used as different color space in our approach.

\section{Hair removal in dermoscopy images}

The existence of hair is a predominant problem in dermoscopy images. In this case, if a skin lesion is covered with hair, the skin lesion segmentation, pattern analysis, and classification tasks will be affected. Thus, an automatic hair removal which preserves the lesion features, while keeping the computational cost low for real-time implementation, is a must. We focus on the on hair removal algorithms, and the hair repairing methods such as harmonic inpainting since this is considered are dominant artifacts on dermoscopy images.

Then, MF filtering of the each image is performed, in the RGB color space in two dimensions. Each output pixel contains the MF value in a $3 \times 3$ neighborhood around the corresponding pixel in the input image. Image was padded with 0's on the edges, so the MF values for points within onehalf the width of the neighborhood $([\mathrm{m} \mathrm{n}] / 2)$ of the edges might appear distorted. A morphological bottom-hat filtering is done on each color space of the filtered image. We then obtain the structuring element. It enhances the contrast of the normal skin with the hair in the dermoscopy images. Conversion of the image to binary image is then performed, based on threshold of 5\%. We perform element-wise multiplication of each multiplies arrays of binarized hair and at each color space of RGB respectively, to obtain NewImage. Similarly, we convert the output image NewImage into a binary image, based on threshold of $2 \%$.

Erosion and dilation by morphological opening on the binary image NewImage with the one square structuring element is performed after. The morphological open operation is an erosion followed by a dilation, using the same structuring element for both operations. We then remove small objects from binary image. To get the hair mask, remove objects with pixels fewer than 90 from binary image. Fill-in the hair pixel regions using the harmonic inpainting technique of [26] from the paper [19] for the area restoration. The flowchart (Fig. 2) of the hair detection, removal, and inpainting steps are shown.

\section{Lesion border detection}

After performing hair removal, lesion border detection follows. Convert the new dermoscopy image to grayscale, then to binary image with a threshold of 50\%. Using Sobel technique [27] perform an edge detection with a fudge factor of $1 \%$ and dilate with a disk strel value of 4 . An image processing technique for identifying borders of the objects in an image is edge detection [17]. Strel [27] is the morphological structuring

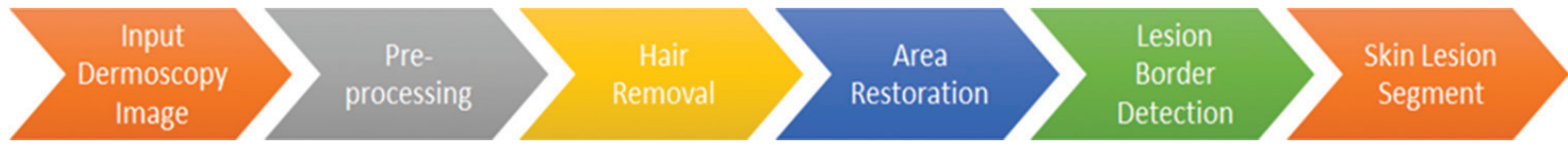

Fig. 1: The flowchart of the skin hair removal method and lesion segmentation 
element used to generate a disk-shaped structuring element, where $\mathrm{R}=4$ identifies the radius. Morphological operations using disk estimate run much faster. Then perform image fill on all holes after getting the perimeter of the lesion. We then perform morphological open operation to remove small objects from the binary image to get the area of the lesion.

\section{RESULTS AND DISCUSSION}

The $\mathrm{PH}^{2}$ dataset is used for benchmarking purposes, to facilitate comparative studies on both segmentation and classification algorithms of dermoscopy images. The dermoscopy image database is acquired at the Dermatology Service of Hospital Pedro Hispano, Matosinhos, Portugal [24]. The dermoscopy images used were under the same conditions through Tuebinger Mole Analyzer system using a magnification of $\times 20$. These images are 8 -bit RGB color with a resolution of $768 \times 560$ pixels.

The image database contains a total of 200 dermoscopy images of melanocytic lesions including 80 common nevi, 80 atypical nevi, and 40 melanomas. The $\mathrm{PH}^{2}$ database consists of medical annotation of every image such as medical segmentation of the lesion, clinical and histological diagnosis, and the evaluation of several dermoscopy criteria such as color, pigment network, dots/globules, streaks, regression areas, and blue-whitish veil

There were available lesion segmentation in binary mask, as a Ground Truth (GT) with the same size of the original image. The GT in the dataset is used to evaluate the performance of our approach. Our approach is implemented in MATLAB 9.2. The method took in an average of $36 \mathrm{~ms}$ to segment and $53.59 \mathrm{~ms}$ for the hair removal including the inpainting. A quantitative evaluation is performed on segmentation by the

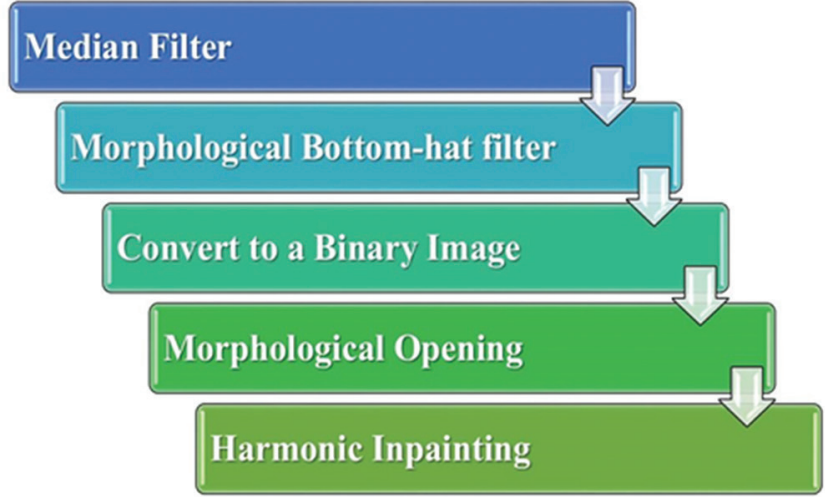

Fig. 2: The flowchart of the hair removal section

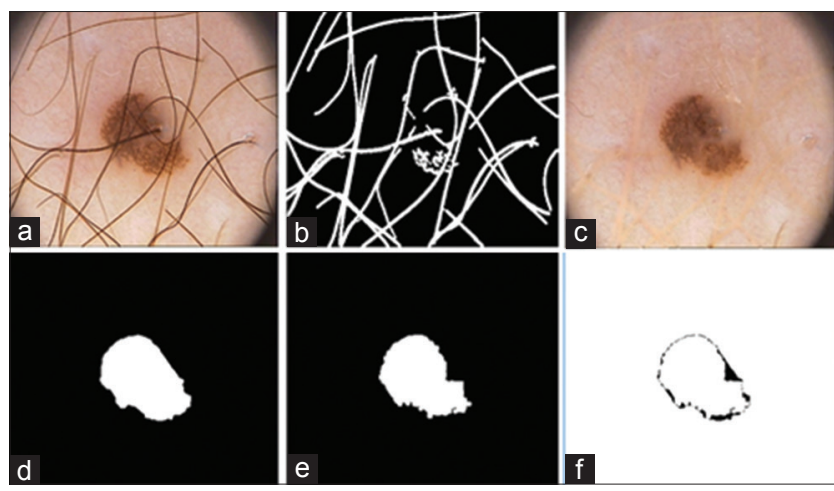

Fig. 3: Lesion border detection results from left to right, top to bottom (a) original dermoscopy image, (b) hair mask, (c) new image without the hair, (d) ground truth (GT) lesion, (e) lesion identified with hair artifacts removed, and (f) lesion identified subtracted with the GT statistical metrics. The border detection outcomes were compared with the reference images or ground truth from [25].

As shown in Fig. 3, the lesion border detection results from top to bottom, respectively. The first image shows the original dermoscopy image, next is the identified hair mask using, next is the new image without the hair, next shows the GT lesion from [24], then the lesion identified with hair, and the last image shows the lesion identified without hair. No or poor lesion detection will occur if hair removal is not performed. As shown in Fig. 4, the first column shows the original dermoscopy image, the second column shows the lesion from the dataset of $\mathrm{PH}^{2}$, and the third column shows the lesion identified using our approach.

The statistical analyses [28] we employed were mean border error (MBE), Hammoude distance (HM), true detection rate (TDR), and the false positive rate (FDR). We denote $N S$ as new segmentation and GT as the GT segmentation from [24]. In MBE, we calculate where NS disagree with GT and are calculated in percentage using eq. 3.

$$
M B E=\frac{\operatorname{area}(N S) \oplus \operatorname{area}(G T)}{\operatorname{area}(G T)} \times 100
$$

The HM metric makes pixel by pixel comparison between $N S$ and GT using eq. 4 .

$$
H M=\frac{\#(N S \bigcup G T)-\#(N S \bigcap G T)}{\#(N S \bigcup G T)}
$$
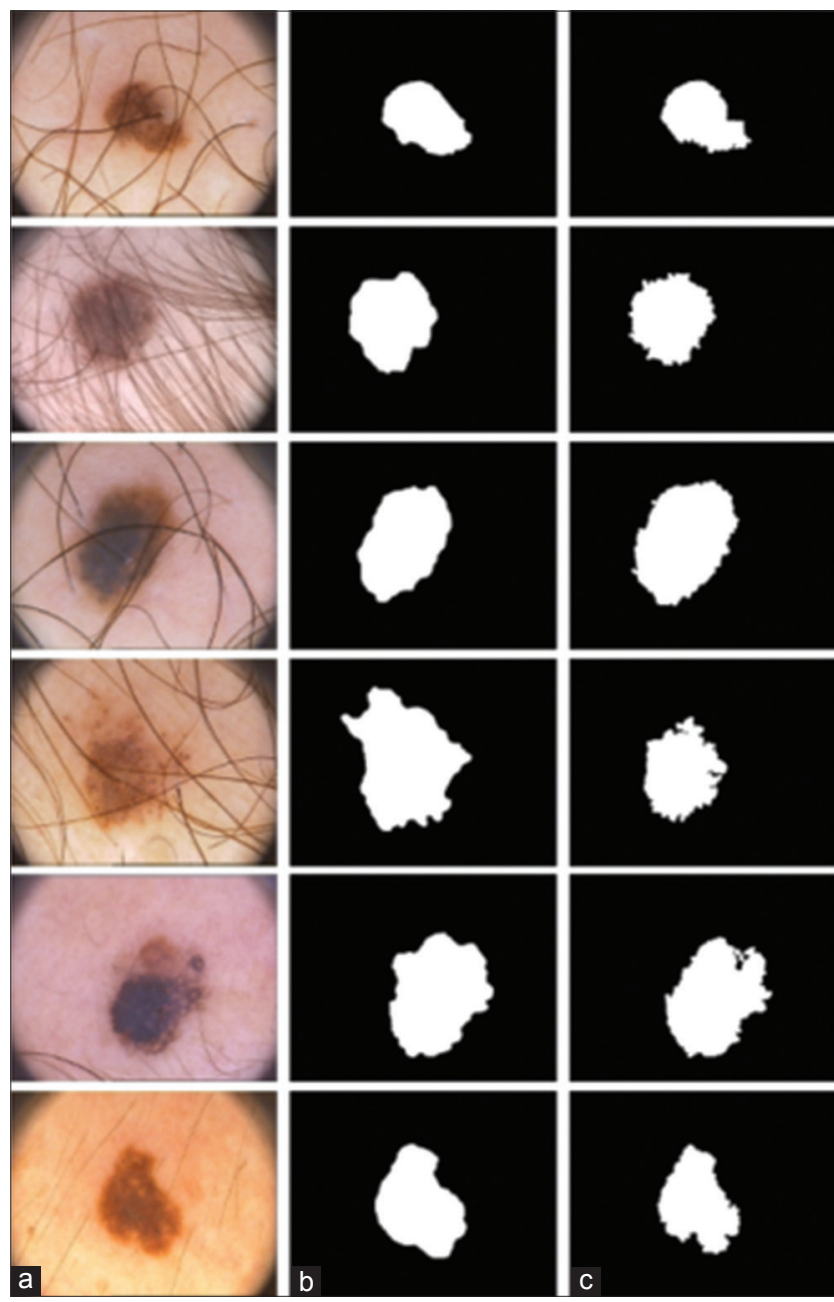

Fig. 4: Lesion border detection results (a) original dermoscopy image, (b) lesion from $\mathrm{PH}^{2}$, (c) lesion border from our approach 
Table 1: Results of performance in four metrics using our approach

\begin{tabular}{ll}
\hline Metric & Results \\
\hline TDR (\%) & 82.31 \\
FDR (\%) & 5.65 \\
MBE (\%) & 15.93 \\
HM (Pixel) & 23.73 \\
\hline
\end{tabular}

TDR: True detection rate, FDR: False-positive rate, MBE: Mean border error, HM: Hammoude

The TDR metric measures the rate of pixels using the new segmented lesion (NS) and the ground truth segmentation (GT) computed using eq. 5 .

$$
T D R=\frac{\#\left(N S \bigcap^{G T)}\right.}{\#(G T)}
$$

The FDR metric measures the rate of pixels using the new segmented lesion (NS) and the ground truth segmentation (GT) computed using eq. 6 .

$$
F D R=\frac{\#\left(N S \bigcap_{\overline{G T})}^{\bar{G}}\right.}{\#(G T)}
$$

The result of the four metric is acceptable to measure the lesion border detection. Table 1 shows the performance of our approach in the dermoscopy images. The border detection difficulty is on detection the difference between the skins of different type, color, or texture. This image segmentation delivers good results based from the preliminary images using dermoscopy images. The problem on the lesion area is sometimes diffused on the skin and is not well separated in the skin. In some images the skin objects are the same color with skin lesions. This gives additional problem and difficulty on border detection. Based on the results as shown in Fig. 3-4, better segmentation of lesion if we perform automatic hair removal.

\section{CONCLUSION}

This paper, we have presented a simple algorithm for lesion border detection of dermoscopy images. In hair removal, we use morphological operators and inpainting. This straightforward approach was shown to be as effective as other methods in detecting, removing, and correcting hair on dermoscopy images. For lesion border detection, we also use morphological operators. This is an important preprocessing step in the identification and classification of melanomas because hair introduces unnecessary features to the image, and lesion border detection is one feature of classifying it. In the future, more image segmentation methods for detecting lesion border on skin images may be explored. Apply deep learning techniques to classify regions of lesion. Skin detection may also be useful, especially looking into the problem of varying skin color for different ethnicities.

\section{ACKNOWLEDGMENT}

The authors would like to thank the anonymous referees for their valuable comments and helpful suggestions. Ms. Salido acknowledges the Commission on Higher Education, in collaboration with the De La Salle University (DLSU) and Aklan State University, for funding support through the Commission on Higher Education K-12 Transition (CHED K-12) Program. Dr. Ruiz is funded by the University Research Coordination Office (URCO) of DLSU.

\section{CONFLICTS OF INTEREST}

All authors declare that they have no conflicts of interest.

\section{REFERENCES}

1. Taylor SC. Skin of color: Biology, structure, function, and implications for dermatologic disease. J Am Acad Dermatol 2002;46:S41-62.
2. Feldman SR, Fleischer AB Jr. McConnell RC. Most common dermatologic problems identified by internists, 1990-1994. Arch Intern Med 1998;158:726-30.

3. Papamichail M, Nikolaidis I, Nikolaidis N, Glava C, Lentzas I, Marmagkiolis $\mathrm{K}$, et al. Merkel cell carcinoma of the upper extremity: Case report and an update. World J Surg Oncol 2008;6:32.

4. Sneyd MJ, Cox B. Melanoma in Maori, Asian, and pacific peoples in New Zealand. Cancer Epidemiol Biomarkers Prev 2009;18:1706-13.

5. Skin Cancer Foundation. Available From: http://www.skincancer.org/ skin-cancer-information/melanoma. [Last retrieved on $2017 \mathrm{Jul}$ 06].

6. Johr RH. Dermoscopy: Alternative melanocytic algorithms-the ABCD rule of dermatoscopy, Menzies scoring method, and 7-point checklist. Clin Dermatol 2002;20:240-7.

7. Argenziano G, Zalaudek I, Corona R, Sera F, Cicale L, Petrillo G, et al. Vascular structures in skin tumors: A dermoscopy study. Arch Dermatol 2004;140:1485-9.

8. Pehamberger $\mathrm{H}$, Steiner $\mathrm{A}$, Wolff $\mathrm{K}$. In vivo epiluminescence microscopy of pigmented skin lesions. I. Pattern analysis of pigmented skin lesions. J Am Acad Dermatol 1987;17:571-83.

9. Nachbar F, Stolz W, Merkle T, Cognetta AB, Vogt T, Landthaler M, et al. The ABCD rule of dermatoscopy. High prospective value in the diagnosis of doubtful melanocytic skin lesions. J Am Acad Dermatol 1994;30:551-9

10. Abbasi NR, Shaw HM, Rigel DS, Friedman RJ, McCarthy WH, Osman I, et al. Early diagnosis of cutaneous melanoma: Revisiting the ABCD criteria. JAMA 2004;292:2771-6.

11. Menzies AM, Haydu LE, Visintin L, Carlino MS, Howle JR, Thompson JF, et al. Distinguishing clinicopathologic features of patients with V600E and V600K BRAF-mutant metastatic melanoma. Clin Cancer Res 2012;18:3242-9.

12. Di Leo G, Fabbrocini G, Liguori C, Pietrosanto A, Sclavenzi M. In: Kayafas E, Loumos V, editors. ELM Image Processing for Melanocytic Skin Lesion Diagnosis Based on 7-Point Checklist: A Preliminary Discussion. Budapest, Hungary: IMEKO; 2004. p. 474-9.

13. Marghoob AA,Scope A, The complexity of diagnosing melanoma. J Invest Dermatol 2009;129:11-13.

14. Abbas Q, Celebi ME, Garca IF. Hair removal methods: A comparative study for dermoscopy images. Biomed Signal Proc Control 2011;6:395-404.

15. Celebi ME, Iyatomi H, Schaefer G, Stoecker WV. Lesion border detection in dermoscopy images. Comput Med Imaging Graph 2009;33:148-53.

16. Erkol B, Moss RH, Stanley RJ, Stoecker WV, Hvatum E. Automatic lesion boundary detection in dermoscopy images using gradient vector flow snakes. Skin Res Technol 2005;11:17-26.

17. Sonka M, Hlavac V, Boyle R. Image Processing, Analysis, and Machine Vision. US: Cengage Learning; 2014.

18. Bertalmio M, Sapiro G, Caselles V, Ballester C. Image in painting. In: Proceedings of the $27^{\text {th }}$ Annual Conference on Computer Graphics and Interactive Techniques. US: ACM Press/Addison-Wesley Publishing Co; 2000. p. 417-24.

19. Drori I, Cohen-Or D, Yeshurun H. Fragment-based image completion. ACM Transact Grap 2003;22:303-12.

20. Shen J, Jin X, Zhou C. Gradient based image completion by solving poisson equation. Adv Multimedia Informat Proc 2005;2005:257-68.

21. Chan TF, Shen J. Nontexture inpainting by curvaturedriven diffusions. J Visual Communicat Image Represent 2001;12:436-49.

22. Barata C, Ruela M. Two systems for the detection of melanomas in dermoscopy images using texture and color features. IEEE Syst J 2013;99:1-15

23. Silveira M, Nascimento J, Marques J, Maral A, Mendona T, Yamauchi S, et al. Comparison of segmentation methods for melanoma diagnosis in dermoscopy images. IEEE J Sel Top Signal Proc 2009;3:3545.

24. Mendonca T, Ferreira PM, Marques JS, Marcal AR, Rozeira J. PH ${ }^{2}$ - a dermoscopic image database for research and benchmarking. Conf Proc IEEE Eng Med Biol Soc 2013;2013:5437-40.

25. Parisotto S, Schnlieb C, MATLAB Codes for the Image Inpainting Problem, GitHub repository. MATLAB Central File Exchange; 2016.

26. Ying-Dong Q, Cheng-Song C, San-Ben C, Jin-Quan L. A fast subpixel edge detection method using Sobel Zernike moments operator. Image Vis Comput 2005;23:11-7.

27. Inc MathWorks, MATLAB: The Language of Technical Computing, Desktop Tools and Development Environment. US1 MathWorks; 2005.

28. Celebi ME, Mendonca T, Marques JS. Dermoscopy Image Analysis. Boca Raton, FL: CRC Press; 2015 